\title{
2-S15-2
}

\section{Current Therapeutic Strategies and New Drug Development in Multiple Myeloma}

\section{Kiyoshi Okazuka}

Multiple myeloma (MM) is a hematological malignancy that originates from bone marrow plasma cells. In Japan, MM is the second most common hematological malignancy and the number of patients are increasing year by year due to the aging population. In addition to the proliferation of monoclonal neoplastic plasma cells, MM presents with a variety of systemic symptoms termed CRAB manifestations, namely bone lesions, hypercalcemia, anemia, and renal involvement.

The selections of anti-myeloma therapy should be based on the patient's condition, such as newly diagnosed multiple myeloma (NDMM), relapsed and/or refractory multiple myeloma (RRMM), the indication for transplantation, and the presence or absence of organ damage due to myeloma. Therefore, the strategy of anti-myeloma therapy should be determined taking account of the patient's individual organ function.

In recent years, those drugs with various mechanisms of action, such as proteasome inhibitors (PI), immunomodulatory drugs (IMIDs), anti-CD 38 monoclonal antibodies, and anti-SLAMF7 antibodies, have emerged and improved the prognosis of MM patients. On the other hand, MM is still difficult to cure, and it relapses repeatedly and gradually becomes resistant to treatments. Therefore, the development of more promising drugs is expected in the future.

In this symposium, the pathology, current treatment strategy, and new drug development status for MM will be outlined 